L. FERNÁNDEZ BERRENDERO

M. ${ }^{a}$ T. VÁZQUEZ OSORIO

F. J. SANZ HOSPITAL

J. A. MURILLO GONZÁLEZ

A. J. PUERTA FONOLLÁ

Departamento de Ciencias Morfológicas I. Facultad de Medicina.

Universidad Complutense. Madrid.

\section{Anatomía vascular de los tendones peroneos en el tobillo}

Vascular anatomy of the fibular tendons of the ankle

\begin{abstract}
Resumen
Estudiamos la vascularización de los tendones peroneos en el tobillo de 26 miembros inferiores de fetos humanos a término mediante disección. Previamente sometemos a los especímenes a inyección de látex teñido por arteria femoral y posteriormente fijamos en formaldehído.
\end{abstract}

Palabras clave: Vascularización. Tendones peroneos. Tobillo.

\section{Summary}

The vascularization of the fibular tendons of the ankle was studied in 26 lower limbs of term human fetuses by dissection. Colorated latex was injected into the femoral artery before specimens were fixed in formaldehyde.

Key words: Vascularization. Fibular tendons. Ankle.

\section{Introducción}

Un aumento en la práctica deportiva y de la expectativa de vida con la patología que conlleva (obesidad, diabetes, arteriosclerosis...) hace que asistamos en los últimos años a un aumento de la patología tendinosa.

Son bien conocidos los trabajos de tendinopatías del Aquiles $^{1,2,3}$. Lesiones que asientan en el tendón del tibial posterior son motivo de publicaciones como causa del pie plano en el adulto ${ }^{4}$, que algunos autores asocian a un defecto en su vascularización ${ }^{5}$.

SOBEL y cols. ${ }^{6}$ realizan un estudio de la vascularización de los tendones peroneos, buscando una etiología vascular al síndrome fisuario de los tendones peroneos, no encontrando relación entre el aumento de edad y la vascularización con respecto al síndrome.

\section{Material y métodos}

Para este trabajo se han utilizado 26 miembros inferiores de fetos humanos a término; una menor dificultad para la obtención de estos especímenes y el hecho de poseer

\section{Correspondencia:}

L. FERNANDEZ BERRENDERO. Departamento de Ciencias Morfológicas I. Facultad de Medicina. Ciudad Universitaria. 28040 Madrid. una vascularización similar a la del adulto ${ }^{7,8,9}$ hizo que optásemos por estos miembros.

Como material de inyección hemos utilizado látex natural teñido con colorantes de la gama Unispers (Ciba-Geigy, S. A.).

Los fetos se conservan en cámara frigorífica hasta el momento de su utilización. Practicamos la disección femoral a nivel de arco crural y canalizamos dicha arteria con un Abbocath del 20-22, inyectando entre 5 y $10 \mathrm{cc}$ de látex coloreado para el relleno del miembro. Inmediatamente sumergimos el espécimen en una solución de formaldehído al $15 \%$ para el proceso de fijación durante un mínimo de 3 semanas. La disección la realizamos con ayuda de gafaslupa de 5 aumentos e instrumental de microcirugía, realizando fotografías de los distintos planos a diversos aumentos.

\section{Resultados}

La vascularización de estos tendones se realiza a través de ramos musculares procedentes de la arteria peronea posterior (A. fibularis) y vasos sinoviales que penetran por un mesotenon basal común a ambos tendones. Este mesotenon experimenta una bifurcación, de manera que cada tendón peroneo lateral dispone de un mesotenon propio (Fig. 1). 


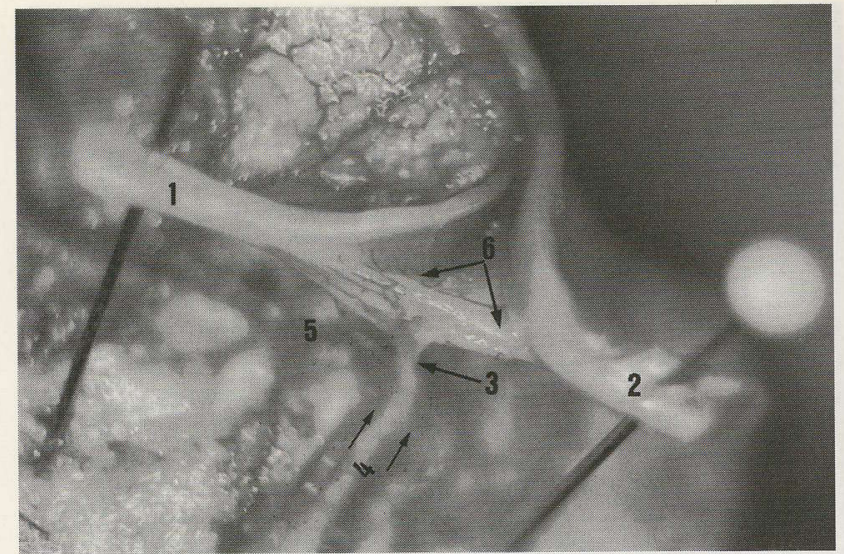

FIG. 1._-Vista anteroexterna del tobillo izquierdo. 1: Tendón peroneo lateral corto (Fibularis brevis). 2: Tendón peroneo lateral largo (Fibularis longus). 3: Mesotenon basal común a ambos peroneos. 4: Cresta de los tendones peroneos (Trochlea peronealis), que determina 2 correderas independientes, la superior para el peroneo lateral corto y la inferior para el peroneo lateral largo. 5: Rama anastomótica de la arteria dorsal del tarso para constituir el arco externo del tarso posterior. 6. Mesotenon propio de los tendones peroneos.

El mesotenon basal es continuo en todo el trayecto de la vaina común, llegando hasta la cresta de los peroneos (Trochlea peronealis) (Fig. 1).

Está ricamente vascularizado con ramas procedentes de la arteria peronea posterior y de las anastomosis de la rama terminal de la arteria peronea posterior, tibial posterior, maleolar externa, plantar externa y ramas terminales de la arteria dorsal del tarso; este arco anastomótico ha sido constante y de él nacen vasos que penetran por el mesotenon basal para distribuirse por ambos peroneos. Los vasos discurren por la cara externa de los tendones peroneos (Figs. 2, 3 y 4).

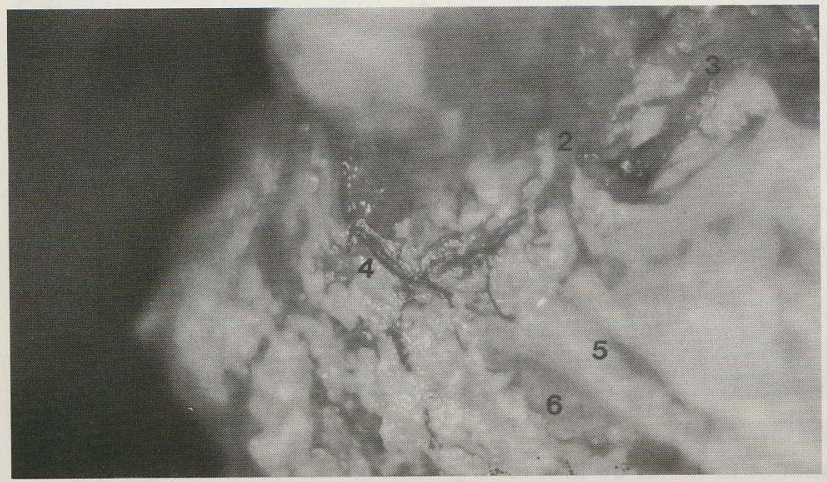

FIG. 2_-Vista externa del tobillo derecho, arco anastomótico externo del tarso posterior. 1: Arteria pedia (A. dorsalis pedis). 2: Arteria dorsal del tarso (A. tarsalis lateralis). 3: Arteria del seno del tarso (A. sinus tarsis). 4: Arco anastomótico externo del tarso posterior. 5: Corredera propia del peroneo lateral corto. 6: Corredera propia del peroneo lateral largo.

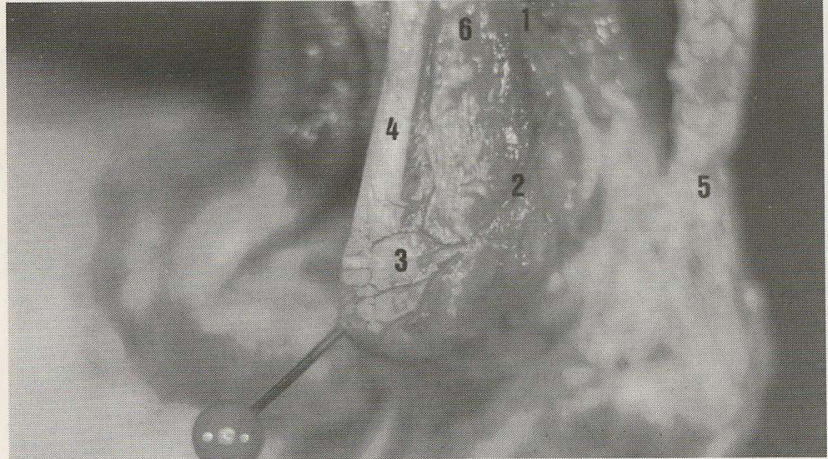

FIG. 3.-Vista posteroexterna del tobillo. 1: Arteria peronea (A. fibularis). 2: Ramos penetrantes vía mesotenon procedentes de la arteria peronea. 3: Distribución vascular, recuerda la disposición de los vasos del mesenterio. 4: Tendón peroneo lateral largo; la vascularización se distribuye por su cara externa. 5: Tendón de Aquiles. 6: Ramas musculares que se anastomosan con vasos procedentes del mesotenon.

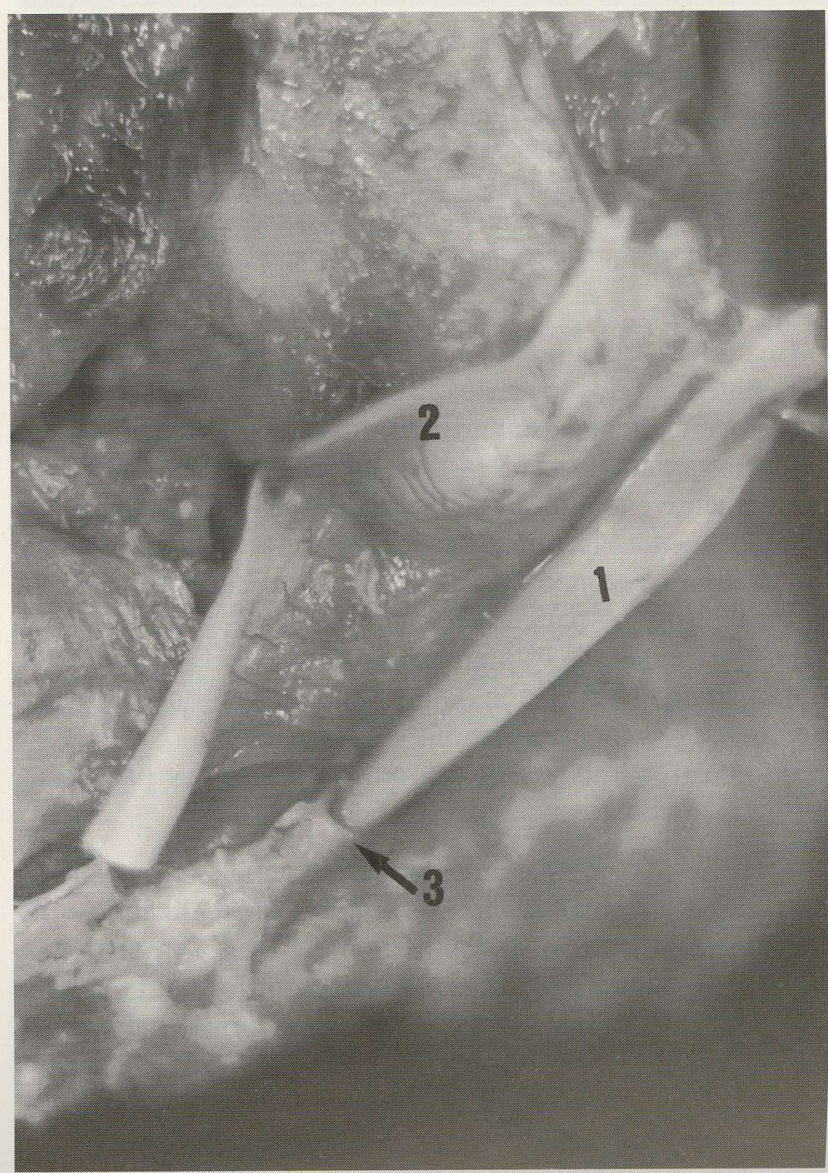

FIG. 4.-Vista externa del tobillo. Seccionados los tendones peroneos. Peroneo largo girado $180^{\circ}$ sobre su eje longitudinal para presentarnos su cara interna. 1: Cara interna del peroneo lateral largo (ausencia de vascularización). 2: Cara externa del tendón peroneo lateral corto (visualizando la vascularización). 3: Corredera propia para el peroneo lateral largo. 


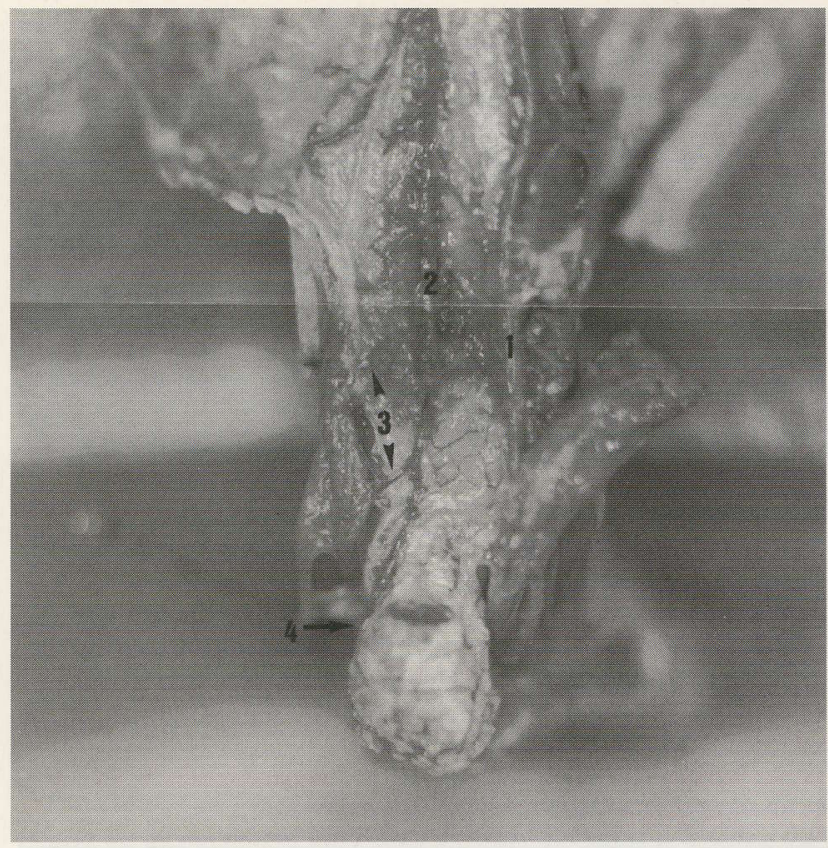

FIG. 5.-Vista posterior de pierna; disposición del patrón vascular más frecuentemente encontrado. 1: Arteria tibial posterior (A. tibialis posterior). 2: Arteria peronea (A. fibularis). 3: Ramos para tendones peroneos. 4: Rama terminal de arteria peronea (arteria calcánea externa que forma el arco anastomótico externo del tarso posterior).

El patrón vascular de los tendones peroneos ha sido constante en todos los casos. Sin embargo, los vasos de los que procede dicha vascularización presentan variaciones.

Observamos (Fig. 5) la disposición vascular más frecuentemente encontrada en la cara posterior de la garganta del pie; la rama terminal de la arteria peronea da ramas a los tendones peroneos y distalmente bordea al peroné por su cara posterior para anastomosarse con ramas de la red dorsal del tarso; de esta anastomosis nacen vasos que penetran, vía mesotenon, en ambos tendones peroneos, distribuyéndose por su cara externa.

En nuestros trabajos encontramos 3 casos que presentan variantes vasculares:

- Caso 1. Corresponde a un caso en que está ausente la rama terminal de la arteria peronea, realizándose la vascularización de los tendones peroneos por ramas procedentes de la arteria tibial posterior; la rama más distal se anastomosa con los vasos dorsales del tarso (Fig. 6).

- Caso 2. Ausencia de la rama terminal de la arteria peronea. Los tendones peroneos se vascularizan con ramas procedentes de la arteria tibial posterior (Fig. 7).

- Caso 3. En este caso la porción más proximal de los tendones peroneos recibe ramas procedentes de la arteria peronea, vascularizándose la porción distal, a través de vasos procedentes de la arteria tibial posterior, que forma el arco anastomótico con los vasos dorsales (Fig. 8).

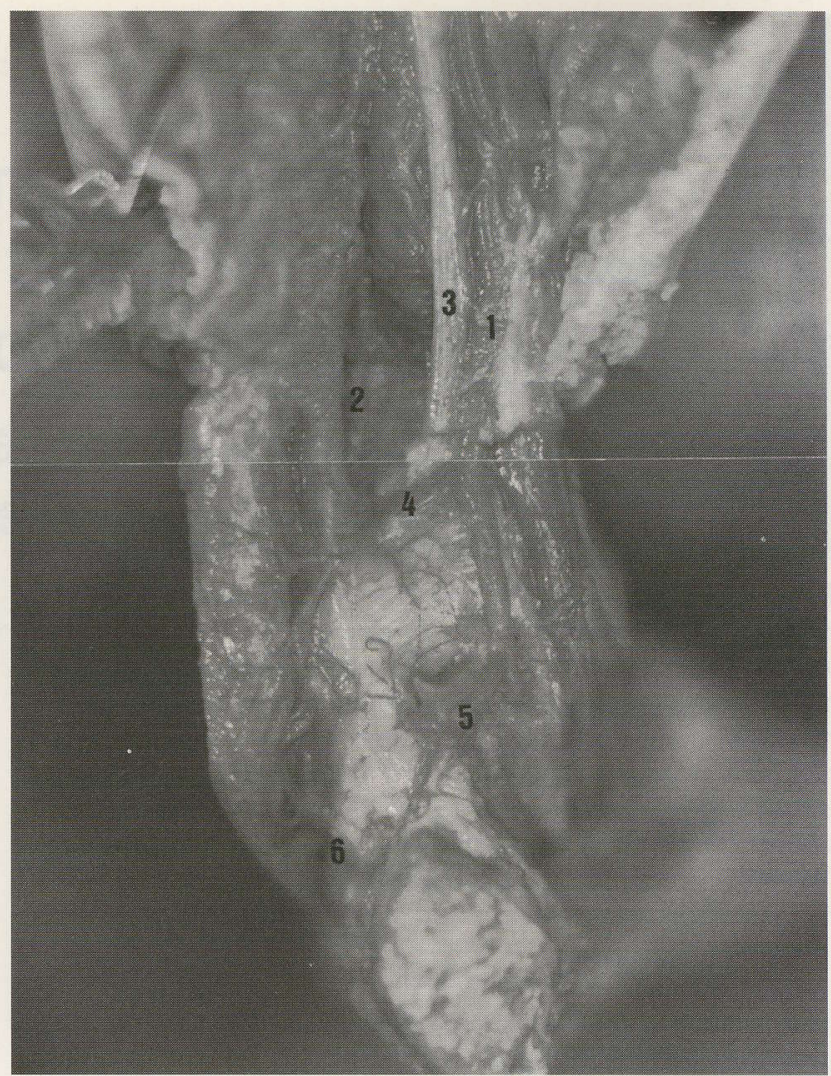

FIG. 6.-Vista posterior de la pierna izquierda. Atrofia segmentaria distal de la arteria peronea. La vascularización de los tendones peroneos es compensada por vasos procedentes de la arteria tibial posterior. 1: Arteria tibial posterior (A. tibialis posterior). 2: Arteria peronea (A. fibularis). 3: Nervio tibial (N. tibialis). 4: Ramas de vascularización de peroneos. 5: Rama anastomótica para constituir el arco externo del tarso posterior.

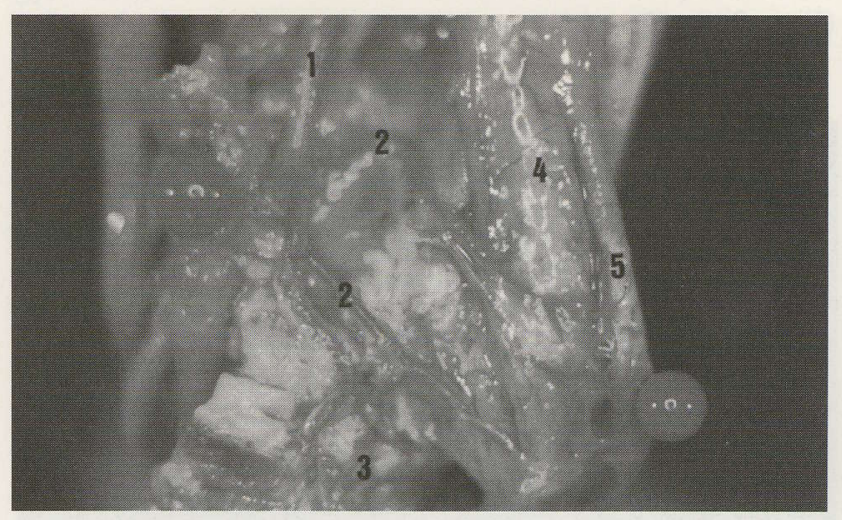

FIG. 7.-Vista posterior de una pierna derecha. Atrofia segmentaria distal de la arteria peronea. Su vascularización la suplen vasos procedentes de la arteria tibial posterior. 1: Arteria tibial posterior (A. tibialis posterior). 2: Ramos procedentes de la arteria tibial posterior que penetran en tendones peroneos a través del paratenon. 3: Ramos anastomóticos para formar el arco externo del tarso posterior. 4: Peroneo lateral corto (T. peroneus brevis). 5: Peroneo lateral largo (T. peroneus longus). 


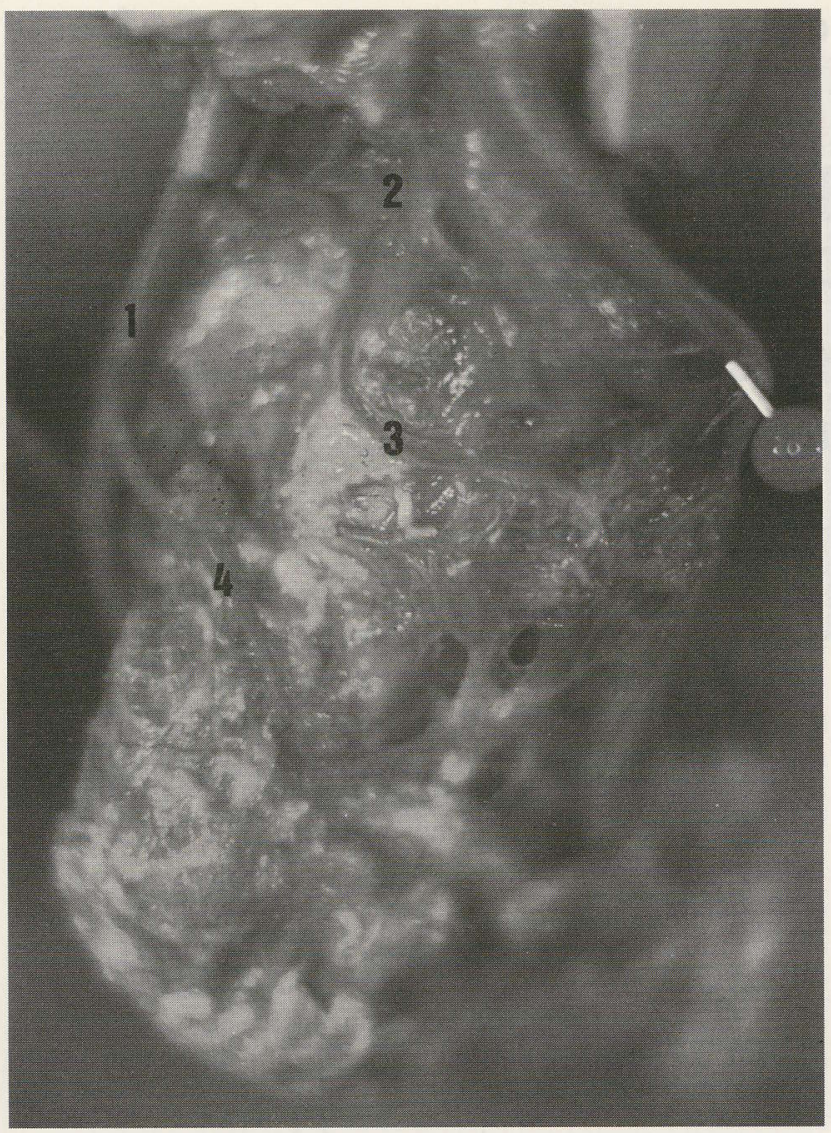

FIG. 8.-Vista posteroexterna del tobillo derecho; atrofia de la porción terminal de la arteria peronea posterior, su vascularización se suple por una rama de la arteria tibial posterior. 1: Arteria tibial posterior (A. tibialis posterior). 2: Arteria peronea (A. fibularis). 3: Ramo terminal de arteria peronea hacia los tendones peroneos. 4: Rama de la arteria tibial para tendones peroneos; forma parte del arco anastomótico externo de tarso posterior.

Estas variantes las valoramos como atrofia parcial y distal de la arteria peronea, que en nuestra serie representarían un $11,1 \%$ del total de los casos estudiados.

\section{Conclusiones}

Los tendones peroneos presentan un patrón constante en su vascularización, independientemente de las variaciones vasculares de los vasos de los que proceda.

Los tendones peroneos disponen de una vascularización proximal procedente de la unión músculo-tendinosa a los que se unen vasos del paratenon que adoptan una disposición similar a la vascularización del mesenterio.

En la vaina común de los tendones peroneos encontramos un mesotenon basal común a ambos que se bifurca para proporcionar un mesotenon propio para cada tendón. Este mesotenon basal llega distalmente hasta la cresta de los peroneos.

Proximal a la cresta de los peroneos encontramos un arco anastomótico que hemos denominado arco anastomótico externo del tarso posterior, del que emergen vasos que penetran por el mesotenon basal para distribuirse por ambos tendones peroneos.

En el trayecto del tobillo los tendones peroneos no presentan zonas avasculares.

\section{Bibliografía}

1. Gómez Castresana F. Roturas del tendón de Aquiles en el deportista. Rev Ortop Traum. 1985;29IB(3):277.

2. Saillant P. La tendinite d'Achille chez le sportif. Science \& Sports. París: Elsevier, 1989;4:327-331.

3. Borrell J. Patología del tendón de Aquiles en el deportista. Avances Traumatológicos. 1989;19-3:111-123.

4. Johnson K. Tibialis posterior tendon rupture. Clin Orthop. 1983 177:145.

5. Frey C. Vascularity of the posterior tibial tendon. $J$ Bone and Joint Surg. 1990;72A(6):884.

6. Sobel M. Microvascular anatomy of the peroneal tendos Foot \& Ankle. 1989;13(8):469-720.

7. Carret JP. Vasculisation arterielle du tendon d'Achilles chez l'homme. Med Chir Pied. 1985;2(4):83-85.

8. Fischer LP, Carret JP. Vascularisation arterielle du ligament rotulien et du tendon d'Achille. Bull Ass Anat. 1976;60:323-334.

9. Sanz Hospital FJ. Vascularización del tendón de Aquiles y de las redes maleolares. Tesis Doctoral. Universidad Complutense. Madrid, 1992. 